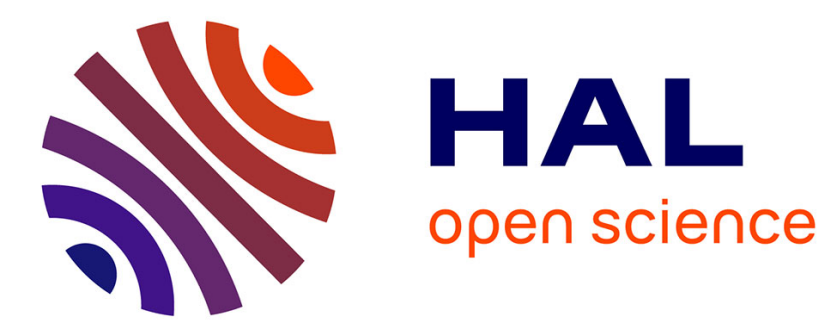

\title{
Distinguishing Resource type in Bpmn Workflows at Simulation Phase
}

Kawtar Ougaabal, Yves Ducq, Grégory Zacharewicz, Said Tazi

\section{To cite this version:}

Kawtar Ougaabal, Yves Ducq, Grégory Zacharewicz, Said Tazi. Distinguishing Resource type in Bpmn Workflows at Simulation Phase. SpringSim 2019 - Spring Simulation Conference, Apr 2019, Tucson, United States. 10.23919/SpringSim.2019.8732850 . hal-02103930

\section{HAL Id: hal-02103930 https://hal.science/hal-02103930}

Submitted on 20 Apr 2020

HAL is a multi-disciplinary open access archive for the deposit and dissemination of scientific research documents, whether they are published or not. The documents may come from teaching and research institutions in France or abroad, or from public or private research centers.
L'archive ouverte pluridisciplinaire HAL, est destinée au dépôt et à la diffusion de documents scientifiques de niveau recherche, publiés ou non, émanant des établissements d'enseignement et de recherche français ou étrangers, des laboratoires publics ou privés. 


\title{
DISTINGUISHING RESOURCE TYPE IN BPMN WORKFLOWS AT SIMULATION PHASE
}

\author{
Kawtar Ougaabal, Yves Ducq \\ University of Bordeaux - Bat A31 \\ Laboratory IMS UMR CNRS 5218 \\ 351 Cours de la Libération, 33405 Talence cedex, \\ FRANCE \\ \{kawtar.ougaabal,yves.ducq\}@u-bordeaux.fr
}

\author{
Grégory Zacharewicz \\ IMT Mines Ales - Laboratory LG2IP \\ Bâtiment M site de Croupillac \\ 7 rue Jules Renard - 30319 Alès cedex \\ France \\ gregory.zacharewicz@mines-ales.fr
}

\author{
Said Tazi \\ University of Pau et Pays de l'Adour \\ Allée du Parc Montaury, 64600 Anglet \\ FRANCE \\ said.tazi@univ-pau.fr
}

\begin{abstract}
The constant search for customer satisfaction and the desire to hold an exclusive position in the market, are two parameters on which the organizations remain vigilant. To keep ahead of the competition, they intend to offer attractive services. Process automation (workflows) remains the most promising solution to save time, money and improve service quality. To better reach this goal, simulation aims to bridge the gap between process requirements and process execution. Several researchers in business process modelling and simulation have focused on control flow and data flow but less attention has been devoted to the resources used. In this paper we propose a finer granularity simulation by taking into consideration the definition and the particularities of each type of resource defined by the Model Driven methodology MDSEA.
\end{abstract}

\section{INTRODUCTION}

The perpetual pursuit for customer satisfaction while maintaining a dominant and influential position in the market, are balancing objectives on which enterprises stay focused. They aim to offer an attractive quality / price ratio that meets customer's expectations while maximizing profit (Schabell 2016). Process automation is a promising solution to save time, money and ensure a controlled service quality. However, business modeling and validation could be very complex. Stakeholders could face many challenges during this course. They may face misunderstanding issues and also face some hardness in analyzing and operationalizing the goal to reach (Dumas et al. 2013).

To overcome these difficulties, Model Driven System Engineering Architecture (MDSEA) has been proposed (Ducq 2014). MDSEA aims to provide a methodology to deal with modelling languages at different abstraction levels (BSM, TIM, TSM, to be detailed further) to support the model transformation from conceptual level into more technical models. One of the main important contributions of this methodology is the definition of three types of resources: IT, Human/organization and Physical means. In our work we started to represent the process at TIM level with business process model. Process modeling 
techniques can be applied as preliminary support by simplifying the representation of the process. However, models provide solely a static abstraction. To go further, simulation can be necessary to assess process's performance at TIM level regarding its dynamicity and behavior (Zacharewicz et al. 2017a). Nevertheless, business process at TIM level needs to be detailed with sufficient description of activities and resources required in order to prepare the simulation. For this purpose, we have extended PyBPMN (Bocciarelli et al. 2016) which is an enhancement of Business process model notation (BPMN) by detailing 3 types of resources defined by MDSEA with their performance attributes. It will support a more complete business process understanding and analysis.

\section{BACKGROUND}

This section presents the concept used in our proposition.

\subsection{Model Driven Engineering (MDE)}

Model Driven Engineering (Schmidt, 2006) is a software development methodology that aims to simplify the entire software engineering process by taking into account the conceptual representations of the system rather than the specifications of the computer technologies alone. To do so, MDE uses models and languages to describe smoothly the need from a high level of abstraction to the concrete solution..

Model driven architecture is defined and proposed by the Object Management Group and it is one of MDE's best-known initiatives. It consists of taking into account separately the business and the technical aspect of an application through models transformations. MDA is a four level architecture leading the transformations from abstract to specific models of a software product. Thanks to MDA models are no longer just a visual or communication element, but a productive one and the backbone of the MDA process. In the same perspective, Model Driven Interoperability (MDI) was initiated within the project INTEROPNoE and is based on MDA (Bourey 2007). This approach considers resolving the interoperability problems by taken them into account from the level of enterprise model rather than the technical level only. The aims of MDI is to decrease the complexity of interoperable software system development by decomposing it into multiple levels through model transformations.

Therefore, the goal of MDE is to increase the communication between all the project stakeholders (Project leader, IT consultant, users...) and to bridge the gap between business requirements and supporting technologies.

\subsection{Model Driven Service Engineering (MDSEA)}

The Model Driven Service Engineering Architecture (MDSEA) is a methodology proposed to support system engineering in classical or Virtual Manufacturing Enterprise (VME) environment. MDSEA is elaborated on the basis of MDA/MDI and aims to overcome their lack of modelling and developing services (Ducq 2014;Zacharewicz et al. 2017b).

In the spirit of model driven approaches, MDSEA proposes a unified methodology to manage modeling languages at various abstraction levels to support service models, service system design and implementation. This approach defines three levels of modelling which link the phases of the service system lifecycle: Business Specific Model (BSM), Technology independent Model (TIM) and Technological Specific Model (TSM).

One of the main contributions in MDSEA is the definition of the three types of service system components (IT, Organization/Human and Physical Means) and their integration at the BSM level (the highest level of modeling). The goal is to ensure that the aspects of the components will be spread out at other levels. 


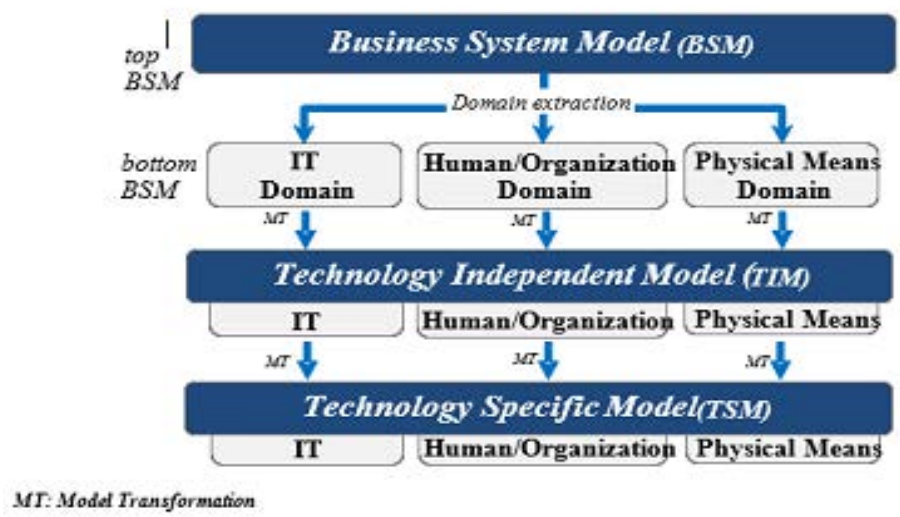

Figure 1: Model Driven System Engineering Architecture (MDSEA).

As shown in Figure 1, MDSEA starts with BSM level to specify models at a highest level of abstraction, describing the interactions of the service system within a single enterprise or a set of enterprises only (Top BSM). The aim of this sub-level is to focus on the statement needed. Based on this initial analysis, the service system will be described according to the different components (Bottom BSM). The goal of this sub-level is to decompose the service system in order to create three main categories of resources. Following a model transformation of the BSM bottom, the model at TIM level is created. This level focuses on describing the operation details of the three components without including any technological specification. Regarding the TSM level, it enhances the model of the TIM level by including implementation details to specify how a system will use a particular type of technology (such as IT infrastructure, Physical means, Specific person...). A detailed explanation of each level in MDSEA is in (Ducq 2014).

\subsection{Enterprise modeling}

Business reengineering and business redesign require a clear understanding of the current status of business operations which will help to make decisions on the changes needed. This is where Enterprise Modelling (EM) can greatly help. EM allows the representation and the description of all enterprise's dimensions or a part of them in a conceptual model at BSM and TIM level of MDSEA (Zacharewicz et al 2017b). It describes operational and decisional structure, processes, resources, etc. In this regard, EM provides the languages and methods that are essential to elaborate enterprise model's to facilitate the understanding of an enterprise system. It helps analyzing, understanding a current status of an enterprise and improving its performance. According to (Kettinger et al, 1997), there are many tools and languages available that allow to describe the different aspects of a system for different business sectors. Among them, SADT (Structured analysis and design technique), IDEF (Integration Definition: IDEF0 ... IDEFx), GRAI model and ARIS (Architecture of Integrated Information System) (Vernadat 1997).

There are different dimensions that can be addressed using enterprise modelling: Processes, Products/Services, Organization, Resources, Information, Environment according to (Rolstadås and Andersen, 2000). In this paper we focus on enterprise processes modelling at TIM level.

\subsection{Business Process Modelling}

Business process model is a structured set of activities or tasks that uses one kind of inputs or more, to produce a specific output (product or service) (Dumas et al. 2013), it describes enterprise processes at TIM level of MDSEA. With the evolution of the market and technology, the business process world has evolved 
rapidly. Processes became much more complex, they can be coordinated from behind, within and beyond traditional boundaries of organizations (Zacharewicz et al. 2017a). That is why business process management is widely used to better manage the lifecycle of processes within an enterprise and business process modelling (BPM) is its foundation (Weske 2007) since it aims to describe the real world process as a mean to understand the model, analyze it and finally implement it.

As enterprise modelling, the scope of BPM can be divided into two categories: organizational design and application design (Dumas et al. 2013). The organizational approach is employed to simplify the complexity of interactions and relationships between activities and its resources regardless of any technical detail. While the application approach is used for process implementation and automation that required the integration of technical specifications.

Various process modelling languages and techniques are available and used in practice (Weske 2007). They can be divided into two different categories: Formal and semi-formal. Semi-formal languages are used to analyze the process from a business perspective to establish its functional requirements, such as Unified Modeling Language activity diagram (UML), Event-driven Process Chain (EPC) and Business Process Modeling and notation (BPMN). On the other side, formal languages are based on rigorous mathematical paradigms such as Petri nets (Van der Aalst 1998). They are used for process automation, process analysis and process simulation. The improvement of the semantics of semi-formal techniques has become (and remains) a vast field of research. This is due to the fact that these languages are the most used in the industry as they are easy to understand, even if the features provided by the formal ones are very useful in terms of improvement of the process.

BPMN is a graphical notation standardized and maintained by the Object Management Group (OMG). The main goal of BPMN is to provide an easily understandable notation by all business stakeholders, from the business analyst who creates the first process drafts to the IT developers responsible for implementing the technology that performs those processes and finally to those responsible to maintain and monitor them. Furthermore, BPMN is easy to learn and is associated to a set of execution languages : BPEL(Business Process Execution Language).

\subsection{Business Process Simulation}

There is an interest to validate process model behavior at TIM level before the implementation phase of the technological solution at TSM level of MDSEA. Indeed all model errors are corrected at this step to avoid, as possible, modifications of running processes in production platform which is a time and money saving. For this aim, we propose in the following to run simulations of BPMN models to observe scenario's performance.

\subsubsection{Recall Process Simulation}

The purpose of simulation is to add a dynamic component to the business process model in order to increase its credibility by clarifying complexities or discovering unknown problems. Moreover, business process simulation allows playing different scenarios by describing the behavior of the system under various alternative actions or decisions (Van der Aalst 2013). Thus, simulation aims to facilitate the process analysis and the process improvement.

However, the effective use of business process simulation is still limited in practice. This is due to the fact that many of the approaches proposed require a mastery of the field, moreover, to guarantee the conformity between the conceptual model and simulation model, it requires a lot of tests, and the tools available in the market are in line with their needs (Bocciarelli et al. 2014). To overcome these issues, a couple of work proposed extensions of BPMN with non-functional requirements. Those propositions could be categorized in two types. The first type proposed a BPMN extension by adding new elements to its meta model like table format, new decorators (Korherr and List 2007; Gagné and Trudel 2009)which alter the specification of the standardize language. The second type proposed a BPMN extension that respects the specification 
provided by the OMG by representing the additional needs using text annotation (Bocciarelli and D’Ambrogio 2011).

Several researches in business process modelling and simulation focused on control flow and data flow and less attention has been devoted to the resources used. The next section will provide a concise review of resource management investigation.

\subsubsection{Related work in business process resources}

In the BPMN 2.0 the description of resources is abstract. In its specification, it is clearly stated that the modelling of organizational structures and resources is out of scope for BPMN (Object Management Group, 2011). Several investigations on the representation of resource specification and resource management were presented. In spite of their relevance, these researches had only shown some aspects of the real use of resources within a workflow case. It is from this perspective that Russel et al. (2005) distinguished 43 Workflow Resource Patterns which define the recurrent requirements in which work items are distributed and executed by resources in workflow systems.

Hence, many researchers had proposed different implementations of those patterns using BPMN language. Awad et al. (2009) proposed an extension of BPMN meta model while respecting the resource allocation patterns requirements. They used the Object Constraint Language to express resource allocation constraints with a user-friendly approach. Stroppi and Chiotti and Villarreal (2011), presented an extension to the BPMN 2.0 meta model to provide support for modeling and visualizing resource perspective requirements by using its extension mechanisms. Their work covers three aspects: resource structure, authorization and work distribution.

The aforementioned contributions are limited to the introduction of BPMN extension in order to enhance its expressive capabilities in terms of resource technical behavior (allocation, distribution...) to choose the WFMS that support the organizational needs.

Other researchers highlighted the need to consider the non-functional requirements of resources rather than the control flow only since the non-functional requirements of a business model are affected by the skills and other factors of the resource assigned. Vasilecas and Laureckas and Rima (2014) proposed an extension of BPMN 2.0 by creating a new graphic element "BPMN comment" to display resources with their parameters such as the role of resource, the availability... They planned to implement this method through the tool BonitaSoft that used BPSIM for simulation. On the other hand, Domingos and Respicio and Martinho (2016) introduced an approach where process engineers could assign resources to a task based on their reliability information and requirements by expressing reliability information in resource definitions and assignments using XML. The reliability of the process is calculated using the Stochastic Workflow Reduction method. The limitation, as addressed by the authors is that the method only covers block structured patterns. In the same perspective, Bocciarelli et al. (2016) proposed an extension of BPMN to represent the real resources used by process instances (human and non-human resources), including their non-functional properties such as performance and reliability. Furthermore, unlike the aforementioned contributions, they took into consideration that a task could be carried out by an atomic resource or a hierarchical composite resources. This approach was implemented using a lightweight BPMN 2.0 extension coupled with the model driven approach to automatically build executable simulation code from BPMN model. Our work aims to improve Bocciarelli et al. (2016) approach by differentiating resource's type, as defined by MDSEA approach at design and simulation type, since each resource has its own characteristics requirements in a business model. 


\section{CONTRIBUTION}

\subsection{Distinguishing resource's type}

As mentioned in section 2.2, one of the main contributions of MDSEA approach is the definition of the three component types of a system (IT, Human, Physical Means) which represent the type of resources within an enterprise. Our work aims to take into consideration this types of resources in the simulation at TSM level to better help the business analyst, IT consultant and users make the best decision regarding the technologies and physical means to be used, as well as the human resources to assign to each task.

Physical means resources are the tangible goods used in a process to carry out its activities (Like machines, robot or any other material handling devices). The efficiency of physical means is measured by its performance, reliability and availability...

Human resources are individuals who work to fulfill their tasks according to their role within a process. Human performance varies from time to time depending on their capability, experience and duration of work...

IT resources are all hardware, software and infrastructure used to accomplish the work required in a process ( Server, Task form, Network...). The description and the measurement of performance of IT resources is well known as Quality of Service (QoS). It could be measured by its bit rate, transmission delay, availability and throughput...

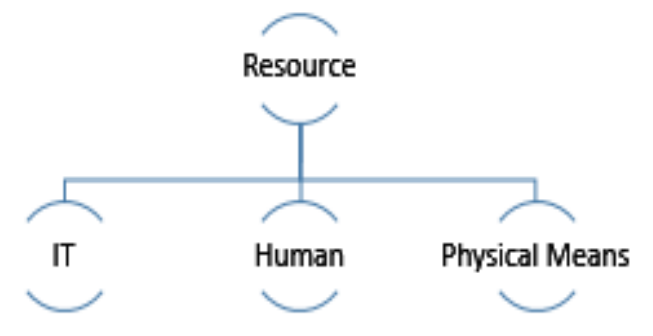

Figure 2: Resources type.

As explained above, each resource has its own definition, its own characterization and its own measurement of efficiency. That is why it is important to distinguish each type at design and simulation time. This work focuses on IT and human resources.

\subsection{PyBPMN extension}

We choose to implement our work by enhancing PyBPMN (Bocciarelli and D'Ambrogio 2011) since it already implements simulation components for performance analysis. As mentioned in section 2.5.2 the extension proposed does not modify BPMN's meta model. And its executable simulation code is implemented according to the BPMN execution semantics (Bocciarelli et al. 2014). These criteria guarantee the standardization of BPMN and the interchangeability of the model. In this section, we will give a brief description of a portion of PyBPMN meta model, more details are mentioned in (Bocciarelli and D’Ambrogio (2011); Bocciarelli et al. 2016)

PyBPMN covers four key areas of non-functional properties : Workload, Performance (service time, response time, throughput), Reliability (occurrence rate of failure, mean time to failure, mean time to repair) and Resource management (specifying non-functional properties for atomic resources (PyPerformer), groups of resources (PySubsystem) and alternative resources (PyBroker).

PyBPMN metamodel consists of a PyElement class that is responsible for the non-functional properties of the business process. Its defined as an abstract class specialized to PyDescriptor and PyBaseResource classes. PyDescriptor class is responsible for linking non-functional attributes with standard BPMN 
elements. PyBaseResource is a class representing the resource and it is specialized in: 1) PyPerformer class represents the performer accomplishing the service requests. 2) PyBroker class is a resource manager that allows you to select a resource from a number of candidate resources.3) PySubsystem class represents a collection of resources required to meet the service request.

In our work, new classes have been added to PyBPMN meta model to distinguish resources types as defined in MDSEA. We propose in Figure 3 to add specialization classes that inherits from PyPerformer. These 3 classes implement PyIT, PyHuman and PyPhysicalMeans. We also implemented new non-functional properties methods as "human experience" for human resources and "loading time" for IT resources in the class PaQualification where performance attributes are implemented.

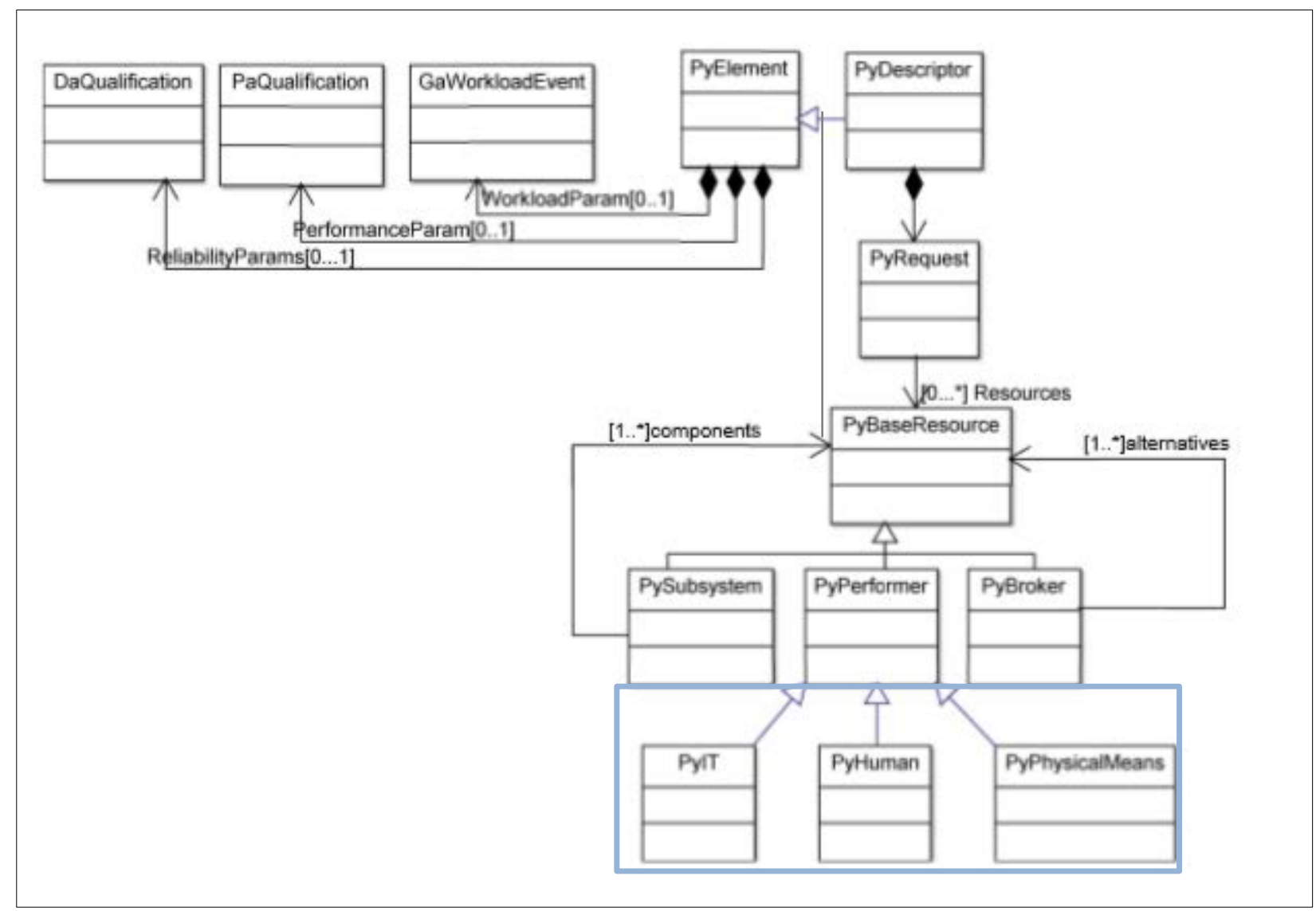

Figure 3: Portion of PyBPMN's class diagram.

\subsection{BPMN extension}

Different task types are identified in BPMN to distinguish the specificities of each type. The commonly used tasks are: Abstract Task, Service task, Human and User task (Polancic 2013). The Abstract Task represents a task with no specialized behavior.The Service task does not require any human interaction, it is completed automatically using a Web service, an automated application... The Manual Task defines the physical work to be performed by human resources without the aid of any software application. It is not managed by any business process engine. On the other hand, User Task represents a task performed by a person with assistance by software applications (Business process management, Enterprise content management...). This task is commonly used in BPMN element it represents a typical workflow task.

User tasks are generally performed via an application's user interface commonly known as task form and are widely used in administrative workflows. Thus, IT resources and human resources are involved to carry out a user task and its efficiency depends on both resources efficiency. Since the aim of our work is to 
provide a finer granularity simulation, we need information about the different components used in a user interface (fields like single line text, checkbox....). The idea is to assign to each component the performance attributes linked to the worker that fill the task form. We want to describe the service time that the user makes to fill each field of a task form (e.g. to fill a "Description" field for a "Check out order" task form, a user can make $5 \mathrm{~min}$ ). We believe that it will help stakeholders (analysts, IT consultant and interface designer) to:

- Choose the best development techniques to implement the interface user: fill some fields automatically, provide some aid that will help the worker fill them...

- Assign the best worker for the task, by taking into consideration the workers experience, their least failures when carrying out similar tasks...

To take into account the information about the different components used in a user interface, we propose an extension of BPMN task diagram by inheriting User Interface class from User Task class. We also use the composition link between User interface class and Field class since a task form is composed of one or multiple fields. This extension is only used for simulation purpose in order to link each field with the worker's service time. No element would be created since we would not corrupt BPMN meta model.

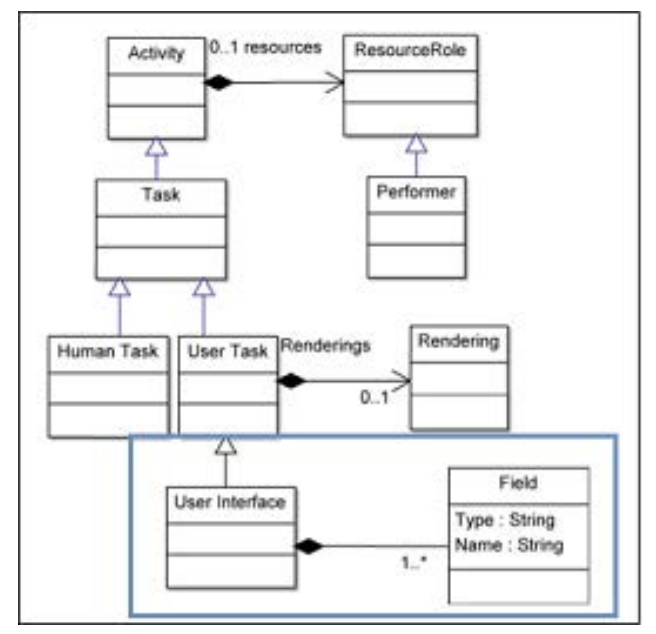

Figure 4: Extending BPMN Task’s class diagram with user interface.

\subsection{Methodology}

The BSM level of MDSEA is not concerned by this modeling phase since it remains at a quite abstract level of description. The resources at BSM are not fully detailed yet. Therefore, we propose to integrate the new resource types defined in sections 3.2 and 3.3 into simulation at TIM level before reaching the TSM level that is close to implementation step.

Figure 6 summarizes the steps and goals of our work. In the first step, the business analyst specifies functional and non-functional requirements including the description of its resources with a BPMN model. After a transformation of BPMN to PyBPMN at first, then a PyBPMN to eBPMN, simulation could be carried out. The simulation after this step aims to propose a finer granularity simulation by taking into consideration the definition and the particularities of each type of resource. This kind of simulation will improves the analysis of the behavior of the process with a set of particular types of resources. Therefore, it helps, with more detailed quantified information, to make the best decision about the workflow design and implementation in: developing techniques/application to use, assigning the right worker to the right task or/and providing training sessions. 


\section{Technology Independent Model TIM}

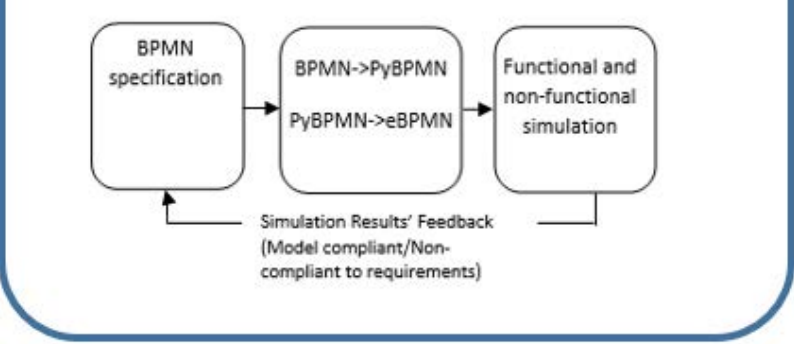

Figure 5: TIM methodology simulation.

The simulation proposed in this approach provides information to analyze the behavior of the workflow system modeled before its implementation. It can quantify the time it takes to call and use different resources involved in a workflow case. As a result, it may be helpful to make the best decisions to simplify or remove some paths of the workflow that are not frequently used; this, in order to reduce the workflow size. Regarding the previous implementation, we obtain a more detailed specification of the resources used by separating human, IT and physical means. We observe that coupling interfaces with resources defines specific performances. For instance, from these results we could strategically reduce the complexity of user forms. We also observe that users that are not experienced with the use of IT can significantly slow down a process.

Our IT partner is now experimenting the use of this methodology to check with their clients the workflow implementation requirements to better meet their needs. In the future, we will define more detailed coupling rules between human $(\mathrm{H})$, IT and physical means (PM) by defining all the possible interactions. For example, we can obtain the following couplings: H.IT.PM, H.IT, IT, IT.PM. In the following we proposed a simplified example to illustrate the BPMN model enriched with resource description details.

\subsection{Simulation Example}

As recalled previously, eBPMN has already proposed attributes to describe non-functional characteristics through a resource. Our work improves this approach by distinguishing resource type. In order to study the feasibility of our work, we use a basic computation of a non-functional characteristic: (experience $\mathrm{x}$ worker's service time) + loading time.

For the sake of simplicity, we present a simple Purchase Request process described in Figure 5. To simplify the figure, we have only shown the text annotations for the task CheckOut order. The text annotation 's syntax is similar to the one proposed by D'Ambrogio (2011), we have just added the new features described in section 3.1 . 


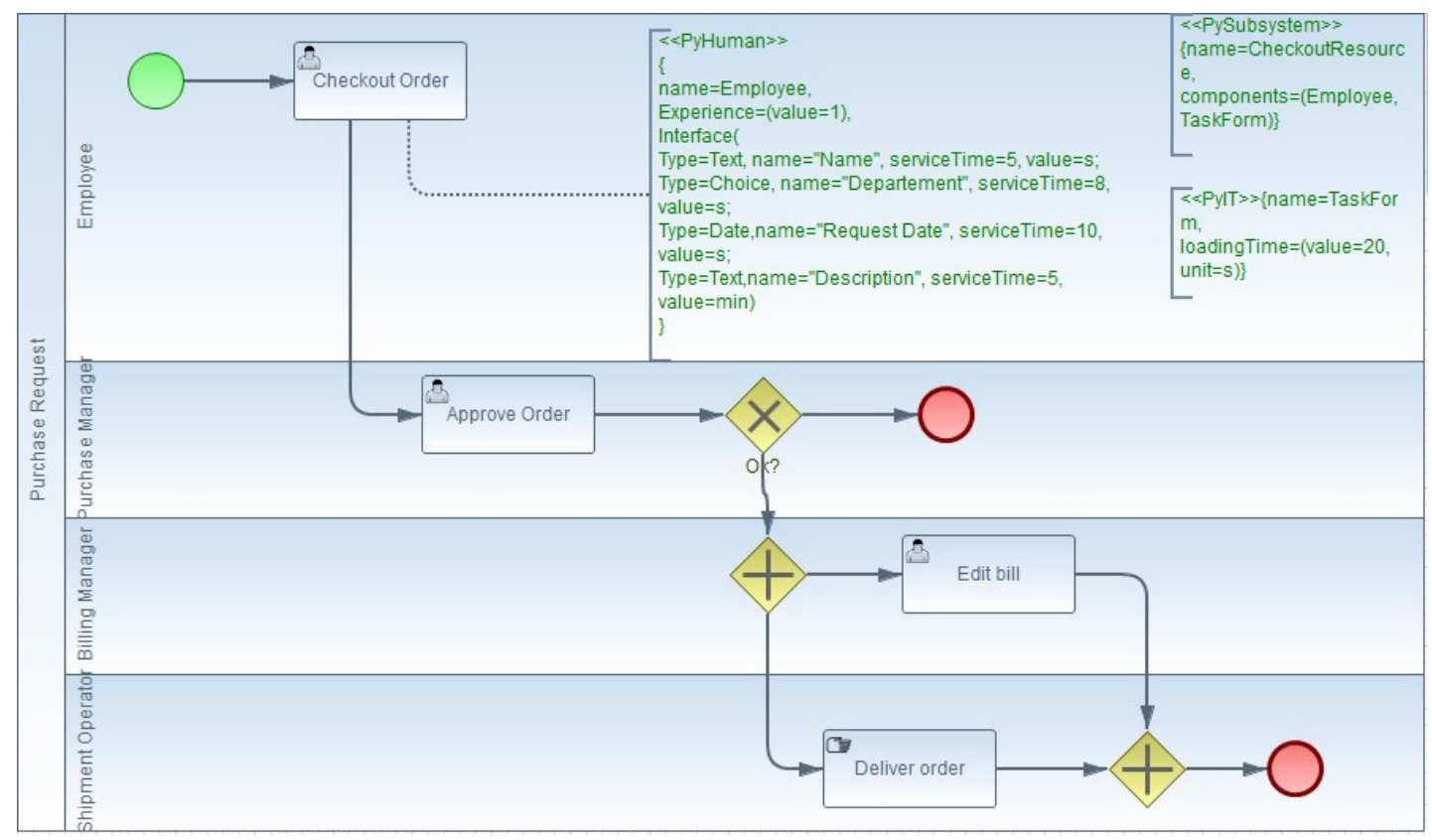

Figure 6: Annotated BPMN.

Here, the User Task is composed by a human resource $(\mathrm{H})$ and IT resource (H.IT). In this example, for the human resource we took into account the user's work service to fill a form and its experience. For the IT resource we considered the loading time attribute depending on the infrastructure to be used (based on the type of network, computer generation, etc.). We detail the different time values in the following table.

Table 1: Worker's service time for each field of Checkout order task form.

\begin{tabular}{|l|l|l|}
\hline \multicolumn{2}{|l|}{ Checkout order Task Form } & Worker's Service Time \\
\hline Name & Type & 5 seconds \\
\hline Name / Last Name & Text & 8 seconds \\
\hline Department & Choice & 10 seconds \\
\hline Request Date & Date & 5 min \\
\hline Description & Text & \\
\hline
\end{tabular}

In our first attempt, by running this simulation, the overall performance was 323 seconds.

When analyzing the result of the simulation, stakeholders were able to see that the Name field, the Department field and Date of Request field could be filled automatically and thus optimize 23 seconds of service time and that could also help users with low experience. Same thing applied for loading time task, the value could be decreased by choosing a better tool such as scrollable component. This deduction can be easily done in this example, but the real workflows studied are composed of thousands of tasks and the simulation helped comparing directly different simulation scenario results.

Thus, the more we progress towards the development of the solution the more complementary and detailed information is necessary to better implement a satisfying process automation and anticipate and reduce the risk to change the implementation of the process during execution phase. 
Ougaabal, Zacharewicz, Ducq and Tazi

\section{CONCLUSION}

This paper proposed an extension of PyBPMN by differentiating resources types defined by MDSEA approach. We introduced the performance for each resource type combination and we proposed a refined simulation at TIM level with the influence of different categories of resources. We have already received the feedback from our IT partner that distinguishing the measurements of each type of resource and carrying out a detailed quantified simulation is helping to bridge the gap between business view and IT view. As a limit, the formulas to compute aggregated performance on different resources coupled with activities are remain fairly simple since we wanted first to test the feasibility of our approach. For future work, we will provide a more advanced computation of activities and resources performances. We will also investigate more in advanced coupling rules of activities and resources.

\section{REFERENCES}

Awad, A. Grosskopf, A. Meyer, A. Weske, M. 2009. "Enabling Resource Assignment Constraints in BPMN". Technical Report.

Bocciarelli, P. D’Ambrogio, A. 2011. “A BPMN Extension for Modeling Non Functional Properties of Business Processes. In Proceedings of the Symposium on Theory of Modeling and Simulation, DEVSTMS '11, pp. 160-168.

Bocciarelli, P. D’Ambrogio, A. Giglio, A. Paglia, E. Gianni, D. 2014. "Empowering Business Process Simulation Through Automated Model Transformations". In Proceedings of the Symposium on Theory of Modeling \& Simulation - DEVS Integrative, DEVS '14, pp. 39:1-39:9.

Bocciarelli, P. D’Ambrogio, A. Giglio and A. Paglia, E. 2016. “A BPMN Extension to Enable the Explicit Modeling of Task Resources". In Proceedings of INCOSE Italia Conference on Systems Engineering

Bourey, J.P. Grangel Seguer, R. and Doumeingts G. Berre, A.J. 2007. "Report on Model Driven Interoperability“. Delivrable DTG2.3, INTEROP Network of Excellence, pp.91

Domingos, D. Respicio, A. Martinho, R. 2016. "Using Resource Reliability in BPMN Processes“. CENTERIS 2016 - International Conference on ENTERprise Information Systems

Ducq, Y. 2014. “An Architecture for Service Modelling in Servitization Context: MDSEA”. Enterprise Interoperability: Research and Applications in the Service-oriented Ecosystem

Dumas, M. La Rosa, M. Mendling, J. and Reijers, H, A. 2013. "Fundamentals of Business Process Management". Springer-Verlag.

Gagné, D. and Trudel, A. 2009. "Time-BPMN". IEEE Conference on Commerce and Enterprise Computing.

Kettinger, W.J. Teng, J.T.C. Guha, S. 1997. "Business process change: a study of methodologies, techniques, and tools “. MIS Quarterly,Vol. 21, No. 1, pp. 55-80.

Korherr, B. List, B. 2007. "Extending the EPC and the BPMN with business process goals and performance measures" in Ninth International Conference on Enterprise Information Systems, Madeira, Portugal pp. 287-294.

Polancic, G. 2013. "BPMN 2.0 Task Types Explained”. White Paper.

Rolstadås, A. Andersen, B. 2000. "Enterprise modeling - Improving global industrial competitiveness" Kluwer Academic, Dordrecht, The Netherlands.

Russel, N. ter Hofsted, A. Edmond, D. Van der Aalst, W.M.P. 2005. "Workflow Resource Patterns"

Schabell, Eric D. 2016 ."Introducing Business Process Management with JBoss BPM". via www.redhat.com/cms/managed-files/mi-jboss-bpm-in-action-ebook-chapter-1-201606-en-2.pdf. Accessed January. 13, 2019 
Schmidt, D.C. 2006. "Model-Driven Engineering" (PDF). IEEE Computer. 39 (2)

Stroppi, L. Chiotti, O. Villarreal, P. 2015. "Defining the resource perspective in the development of processes-aware information systems". Information and Software Technology, Volume 59, pp.36-108

Zacharewicz, G. Pirayesh-Neghab, A. Seregni, M. Ducq, Y. Doumeingts, G. 2017. "Simulation-Based Enterprise Management Model Driven from Business Process to Simulation". Guide to SimulationBased Disciplines

Zacharewicz, G., Diallo, S., Ducq, Y. et al. Model-based approaches for interoperability of next generation enterprise information systems: state of the art and future challenges, Inf Syst E-Bus Manage (2017) 15: 229.

Van der Aalst, W. M. P. 2013. "Business process simulation survival guide. In Handbook on Business Process Management", pages 337-370.

Vasilecas, O. Laureckas, E. Rima, A. 2014. "Analysis of Using Resources in Business Process Modeling and Simulation“. Applied Computer Systems, 16(1), pp-19-25

Vernadat F.B. 1997. "Enterprise Modelling Languages". Enterprise Engineering and Integration. pp.212224

Weske, M. 2007. "Business Process Management: Concepts, Languages, Architectures”. Springer, Berlin, Heidelberg.

\section{AUTHOR BIOGRAPHIES}

KAWTAR OUGAABAL is a Nintex consultant at Exakis (France) and a $\mathrm{PhD}$ candidate at the University of Bordeaux (France). Her thesis focuses on the business process modelling, business process simulation and model transformation. Her email address is kawtar.ougaabal@u-bordeaux.fr.

GREGORY ZACHAREWICZ is Full Professor at IMT - Mines Alès (France). His research interests include discrete event M\&S, distributed simulation (HLA), model driven approaches, semantics aspects, and enterprise modeling. He focused recently on Social-Organizational M\&S and Interoperability. He is involved in several M\&S conferences and journals. He has been participating in a number of French, European and transatlantic M\&S projects. His email address is gregory.zacharewicz@mines-ales.fr

YVES DUCQ is Full Professor at University of Bordeaux (France) from September 2018. He is working on Performance Measurement, Enterprise Modeling and Production Management since fifteen years and has published more than 20 papers in books and international journals and more than 70 papers in international conferences. He is responsible of the research group dedicated to production engineering sciences (GRAI research Group) at IMS laboratory. His email address is yves.ducq@u-bordeaux.fr

SAID TAZI is Senior Lecturer at University of Pau (France) since September 1991. He is working on Performance Measurement, Enterprise Modeling and Production Management since ten years and has published 10 papers in international conferences. His email address is said.tazi@univ-pau.fr 